\title{
Interpolation by Bivariate Splines on Crosscut Partitions
}

\author{
G. Nürnberger, O. V. Davydov, G. Walz \& F. Zeilfelder
}

Nr. $\quad 227 / 1997$ 


\title{
Interpolation by Bivariate Splines on Crosscut Partitions
}

\author{
G. Nürnberger, O. V. Davydov, G. Walz, and F. Zeilfelder
}

\begin{abstract}
We give a survey of recent methods to construct Lagrange interpolation points for splines of arbitrary smoothness $r$ and degree $q$ on general crosscut partitions in $\mathbb{R}^{2}$. For certain regular types of partitions, also results on Hermite interpolation sets and on the approximation order of the corresponding interpolating splines are given.
\end{abstract}

\section{Introduction}

We consider bivariate spline spaces of the following type. Let a convex compact region $\Omega \in \mathbb{R}^{2}$ be given, which is subdivided by a finite number of straight lines (crosscuts) into convex subregions $\{T\}$, called a partition $\Delta$ of $\Omega$. The space of all functions $s \in C^{r}(\Omega)$, whose restriction to each $T$ is a bivariate polynomial of degree $q$, is denoted as the spline space $S_{q}^{r}(\Delta)$.

In Section 2, a construction method for point sets $\left\{z_{1}, \ldots, z_{\operatorname{dim} S_{q}^{r}(\Delta)}\right\}$ on a general partition $\Omega$, which admit unique Lagrange interpolation w.r.t. $S_{q}^{r}(\Delta)$, is described.

The construction of Hermite interpolation sets (which can be considered as limits of Lagrange interpolation sets) for certain rectangular types of partitions $\Delta$, denoted as $\Delta^{1}$ resp. $\Delta^{2}$ partitions, is given in Section 3 . Here, $\Delta^{1}$ denotes the partitions where to each subrectangle the same diagonal is added, while $\Delta^{2}$ means that both diagonals are added.

We also give results on the approximation order of the interpolating spline function. The approximation order for $S_{q}^{r}\left(\Delta^{1}\right)$ equals $q+1$ (which is optimal), if $q \geq 3.5 r+1, r \geq 1$, and $q$, if $r=1$ and $q=4$. For $S_{q}^{1}\left(\Delta^{2}\right)$, we get the optimal order $q+1$, if $q \geq 4$, and the order $q$, if $q=2,3$. 


\section{Construction of Lagrange İnterpolation Sets for Crosscut Partitions}

Let $\Omega \subset \mathbb{R}^{2}$ be a convex compact domain. Any finite set of straight lines (called crosscuts) $l_{1}, \ldots, l_{M}$ having nonempty intersections with the interior of $\Omega$, produces a partition $\Delta$ of $\Omega$ into convex compact subregions (called cells) with pairwise disjoint interiors. We denote by $\mathcal{T}$ the set of all cells. The straight line boundaries of each cell are called edges and their endpoints. vertices. Let $\left\{v_{1}, \ldots, v_{L}\right\}$ be the set of all interior vertices of the partition $\Delta$. Thus, each $v_{i}$ is an intersection point of two or more crosscuts which lies in the interior of $\Omega$.

The space of polynomial splines of degree $q$ and smoothness $r, 0 \leq r<q$, with respect to the partition $\Delta$ is defined by

$$
S_{q}^{r}(\Delta):=\left\{s \in C^{r}(\Omega):\left.s\right|_{T} \in \tilde{\Pi}_{q}, T \in \mathcal{T}\right\}
$$

where

$$
\tilde{\Pi}_{q}:=\operatorname{span}\left\{x^{i} y^{j}: i \geq 0, j \geq 0, i+j \leq q\right\}
$$

is the space of bivariate polynomials of total degree $q$.

The dimension of $S_{q}^{r}(\Delta)$ was determined by Chui \& Wang [6].

We consider the following problem. Determine points $z_{1}, \ldots, z_{N}$ in $\Omega$, where $N=\operatorname{dim} S_{q}^{r}(\Delta)$, such that for any $f \in C(\Omega)$, the Lagrange interpolation problem $s\left(z_{i}\right)=f\left(z_{i}\right), i=1, \ldots, N$, has a unique solution $s$ in $S_{q}^{r}(\Delta)$. Such sets $\left\{z_{1}, \ldots, z_{N}\right\}$ are called Lagrange interpolation sets for $S_{q}^{r}(\Delta)$. (Sometimes we say that $z_{1}, \ldots, z_{N}$ are Lagrange interpolation points for $S_{q}^{r}(\Delta)$.)

First we describe bases and interpolation points for three types of subspaces of $S_{q}^{r}(\Delta)$ and then show how these points can be combined into an interpolation set for the whole space.

A. The space of bivariate polynomials $\tilde{\Pi}_{q}$. It is well known that $\operatorname{dim} \tilde{\Pi}_{q}=$ $\left(\begin{array}{c}q+2 \\ 2\end{array}\right)$, and a basis of $\tilde{\Pi}_{q}$ is given by

$$
\left\{x^{i} y^{j}: i \geq 0, j \geq 0, i+j \leq q\right\} .
$$

Interpolation sets for $\tilde{\Pi}_{q}$ can be obtained in the following way (see, for example, Nürnberger [11]). Let $\gamma_{0}, \ldots, \gamma_{q}$ be distinct parallel lines and $z_{i, 0}, \ldots, z_{i, q-i}$ distinct points on $\gamma_{i}, i=0, \ldots, q$ (see Fig. 2.1). Then

$$
\left\{z_{0,0}, \ldots, z_{0, q}, \ldots, z_{q-1,0}, z_{q-1,1}, z_{q, 0}\right\}
$$

is a Lagrange interpolation set for $\tilde{\Pi}_{q}$, and the points are called interpolation points of type $A$.

B. The space of truncated power functions. Let $l$ be any crosscut dividing $\Omega$ into two subdomains $\Omega_{0}$ and $\Omega_{1}$. We consider the following space:

$$
T_{q}^{r}:=\left\{s \in C^{r}(\Omega):\left.s\right|_{\Omega_{0}} \equiv 0,\left.s\right|_{\Omega_{1}} \in \tilde{\Pi}_{q}\right\}
$$




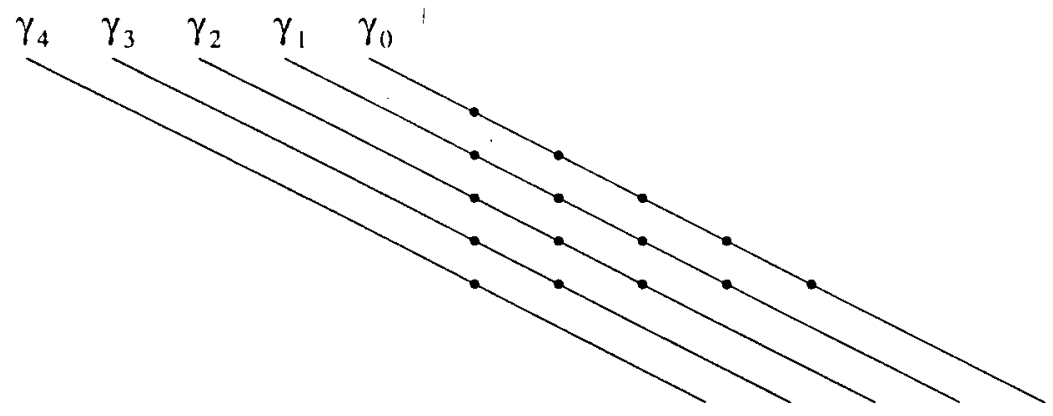

Fig. 2.1. Interpolation points of type A.

Suppose

$$
l=\left\{(x, y) \in \mathbb{R}^{2}: a x+b y+c=0\right\}
$$

such that

$$
a x+b y+c>0, \quad(x, y) \in \operatorname{int} \Omega_{1} .
$$

A basis for $T_{q}^{r}$ is given by truncated powers multiplied with polynomials,

$$
\left\{(a x+b y+c)_{+}^{r+1} x^{i} y^{j}: i \geq 0, j \geq 0, i+j \leq q-r-1\right\},
$$

where

$$
w_{+}^{k}:= \begin{cases}w^{k}, & w \geq 0 \\ 0, & w<0\end{cases}
$$

Therefore,

$$
\operatorname{dim} T_{q}^{r}=\left(\begin{array}{c}
q-r+1 \\
2
\end{array}\right) .
$$

In order to determine interpolation sets for $T_{q}^{r}$, we choose $q-r$ parallel lines $\gamma_{0}, \ldots, \gamma_{q-r-1}$ intersecting $l$ and put $q-r-i$ distinct points $z_{i, 0}, \ldots, z_{i, q-r-1-i}$ on $\gamma_{i} \cap\left(\Omega_{1} \backslash l\right), i=0, \ldots, q-r-1$ (see Fig. 2.2). Then

$$
\left\{z_{0,0}, \ldots, z_{0, q-r-1}, \ldots, z_{q-r-2,0}, z_{q-r-2,1}, z_{q-r-1,0}\right\}
$$

is a Lagrange interpolation set for $T_{q}^{r}$, and the points are called interpolation points of type $B$.

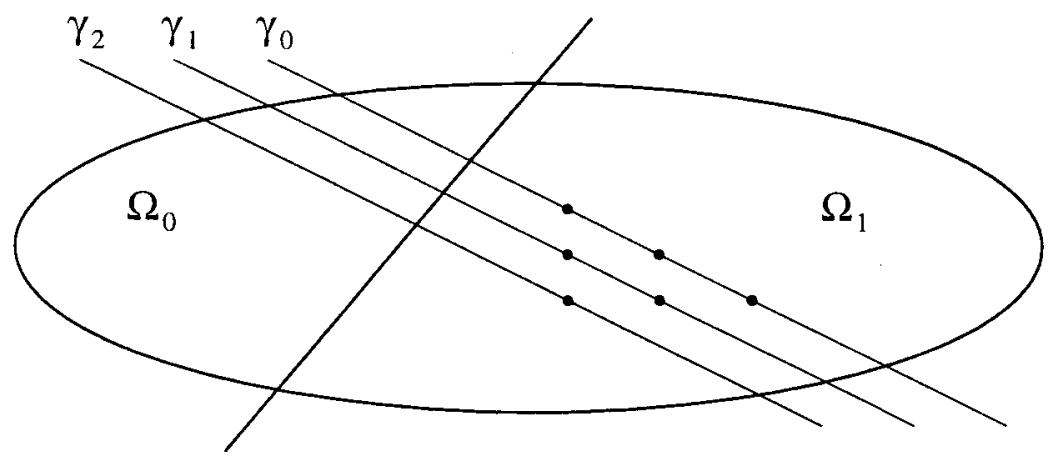

Fig. 2.2. Interpolation points of type B. 
C. The space of cone splines. Let $v$ be any interior vertex such that $m$ crosscuts $l_{1}, \ldots, l_{m}$ intersect at $v$. Thus, $2 m$ rays originate at $v$. We take $m$ consecutive rays $r_{1}, \ldots, r_{m}$ (so that all $m$ crosscuts are involved) and divide $\Omega$ into $m$ subdomains $\Omega_{0}, \Omega_{1}, \ldots, \Omega_{m-1}$ as in Fig. 2.3.

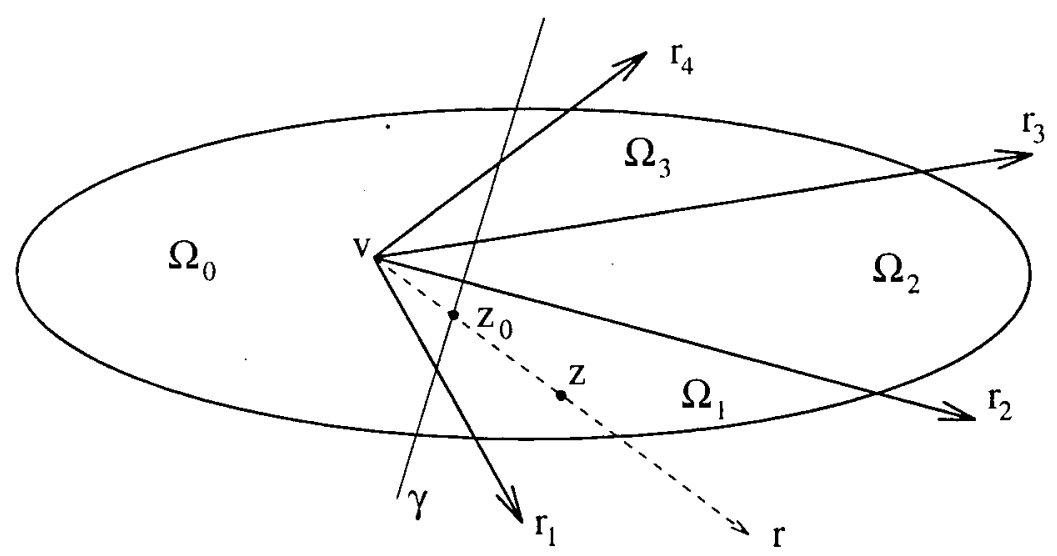

Fig. 2.3.

The space of cone splines $K_{q}^{r}$ is defined as follows.

$$
K_{q}^{r}:=\left\{s \in C^{r}(\Omega):\left.s\right|_{\Omega_{0}} \equiv 0,\left.s\right|_{\Omega_{i}} \in \tilde{\Pi}_{q}, i=1, \ldots, m-1\right\} .
$$

Thus, all splines in $K_{q}^{r}$ are zero outside the cone $\bigcup_{i=1}^{m-1} \Omega_{i}$. We now define a basis for $K_{q}^{r}$. We choose a line $\gamma$ which intersects all the rays $r_{1}, \ldots, r_{m}$, with $v \notin \gamma$ (see Fig. 2.3). For $n=q, q-1, \ldots$, we consider the univariate spline spaces $\left.K_{n}^{r}\right|_{\gamma}$ on $\gamma$ such that $\left.\operatorname{dim} K_{n}^{r}\right|_{\gamma}>0$. Then we extend each univariate $B$-spline $B$ in $\left.K_{n}^{r}\right|_{\gamma}$ to a function in $K_{n}^{r}$ as follows. Let $B(z) \equiv 0, z \in \Omega_{0}$. For each ray $r$ in $\Omega \backslash \Omega_{0}$ passing through $v$, we define $B$ to be the univariate truncated power function $t_{+}^{n}$ multiplied with an appropriate constant. More precisely, let $z \in \Omega \backslash \Omega_{0}$ and let $z_{0}$ be the intersection point of $\gamma$ and the line through $v$ and $z$ (see Fig. 2.3). Then $B(z):=B\left(z_{0}\right)|z-v|^{n} /\left|z_{0}-v\right|^{n}$. All the bivariate splines in $K_{n}^{r}, n=q, q-1, \ldots$, obtained in this way (called cone splines), form a basis of $K_{q}^{r}$ (see Chui \& Wang [6] and Dahmen \& Micchelli [7]). Therefore,

$$
\operatorname{dim} K_{q}^{r}=\left.\sum_{n \leq q} \operatorname{dim} K_{n}^{r}\right|_{\gamma}
$$

We now describe interpolation points for $K_{q}^{r}$. Let $\gamma_{0}, \ldots, \gamma_{p}$ be parallel lines which intersect the rays $r_{1}, \ldots, r_{m}$ and do not pass through the vertex $v$, where $p$ is determined by the conditions $\left.\operatorname{dim} K_{q-p}^{r}\right|_{\gamma}>0$ and $\left.\operatorname{dim} K_{q-p-1}^{r}\right|_{\gamma}=0$. We choose $\left.\operatorname{dim} K_{q-i}^{r}\right|_{\gamma}$ points on $\gamma_{i} \cap\left(\Omega \backslash \Omega_{0}\right), i=0, \ldots, p$, so that these points satisfy Schoenberg-Whitney condition for the univariate spline space $\left.K_{q-i}^{r}\right|_{\gamma_{i}}$ (see Fig. 2.4, a)). 


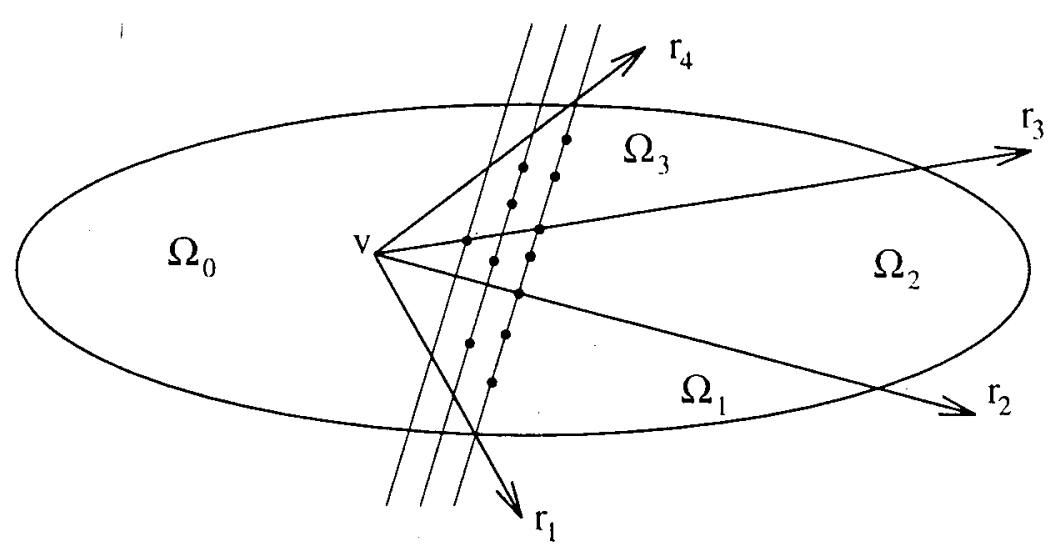

a)

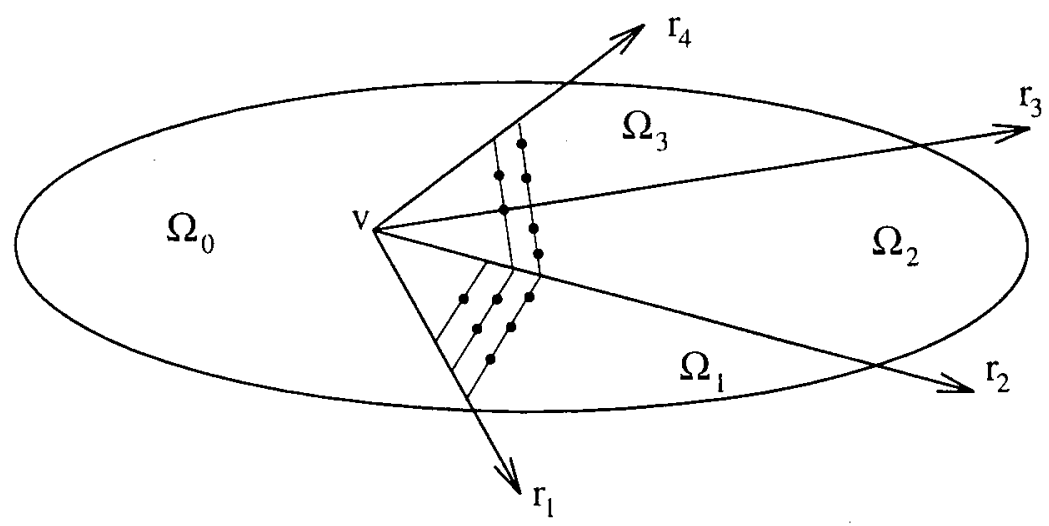

b)

Fig. 2.4. Interpolation points of type C.

In other words, we take a point in a support of each univariate $B$-spline in $\left.K_{q-i}^{r}\right|_{\gamma_{i}}$. It was shown by Nürnberger \& Riessinger [14] that each set of points obtained in this way admits unique Lagrange interpolation from $K_{q}^{r}$. We call them interpolation points of type $C$.

A more general scheme of constructing interpolation points of type $\mathrm{C}$ (see Fig. 2.4, b)) was also proposed in [14]. The cone formed by $r_{1}$ and $r_{m}$ can be divided into several subcones. Then the points are chosen on line segments inside the subcones according to some Schoenberg-Whitney type conditions for the spaces $K_{n}^{r}$ restricted to the line segments as in Fig. 2.4, b). (For details see [14].) Another configuration of interpolation points of type $\mathrm{C}$ was given by Adam [1], where the points are lying on certain rays.

Now we are able to describe a basis for $S_{q}^{r}(\Delta)$ which is due to Chui \& Wang [6] and Dahmen \& Micchelli [7].

Theorem 2.1. A basis of $S_{q}^{r}(\Delta)$ is given by the following functions: 
A. Polynomial basis functions $x^{i} y^{j}, i \geq 0, j \geq 0, i+j \leq q$.

B. Truncated power functions, for each crosscut $l_{i}, i=1, \ldots, M$.

C. Cone splines, as described above, for each interior vertex $v_{i}, i=1, \ldots, L$.

We note that there is some freedom in choosing basis functions in B. and C. of Theorem 2.1 since there are two possible spaces of truncated powers $T_{q}^{r}$ with respect to a given crosscut, and also the rays which define cone splines can be choosen differently. On the other hand, the interpolation points of type A, B and C cannot be freely combined to obtain a Lagrange interpolation set for $S_{q}^{r}(\Delta)$. A method to assign a type $\mathrm{A}, \mathrm{B}$ or $\mathrm{C}$ to each cell of the partition so that the combination of corresponding interpolation points on the cells is an interpolation set for the whole spline space was proposed by Nürnberger \& Riessinger $[13,14]$ for rectangular partitions with diagonals and extended to arbitrary crosscut partitions by Adam [1]. Their construction depends upon an order of the cells which is the natural ordering with respect to rows and columns in the case of rectangular partitions. We now describe this order in the general case of crosscut partitions (see Adam [1]).

The lexicographical order of the points in $\mathbb{R}^{2}$ is defined as follows. Given two points $z^{\prime}=\left(x^{\prime}, y^{\prime}\right), z^{\prime \prime}=\left(x^{\prime \prime}, y^{\prime \prime}\right) \in \mathbb{R}^{2}$ we say that $z^{\prime} \leq z^{\prime \prime}$ if

$$
x^{\prime}<x^{\prime \prime} \text { or }\left(x^{\prime}=x^{\prime \prime} \text { and } y^{\prime} \leq y^{\prime \prime}\right) \text {. }
$$

As usual, $z^{\prime}<z^{\prime \prime}$ if $z^{\prime} \leq z^{\prime \prime}$ and $z^{\prime} \neq z^{\prime \prime}$. For any compact set $K \subset \mathbb{R}^{2}, \quad m(K) \in$ $\mathbb{R}^{2}$ denotes the minimal point of $K$ with respect to the lexicographical order.

The total order of cells $T \in \mathcal{T}$ of the partition $\Delta$ is defined as follows. In the case when $m\left(T^{\prime}\right)<m\left(T^{\prime \prime}\right)$ (in lexicographical order), $T^{\prime}, T^{\prime \prime} \in \mathcal{T}$, we set $T^{\prime}<T^{\prime \prime}$. In the case when several cells have the same minimal point $m$, they are situated to the right of $m$ and separated from each other by ray segments originating at $m$. Therefore, they can be ordered either clockwise or counterclockwise. We choose every time one of these two orders according to the following rule:

Case 1. $m=m(\Omega)$ or $m \in$ int $\Omega$. The cells $\{T \in \mathcal{T}: m(T)=m\}$ are ordered counterclockwise (see Fig. 2.5).
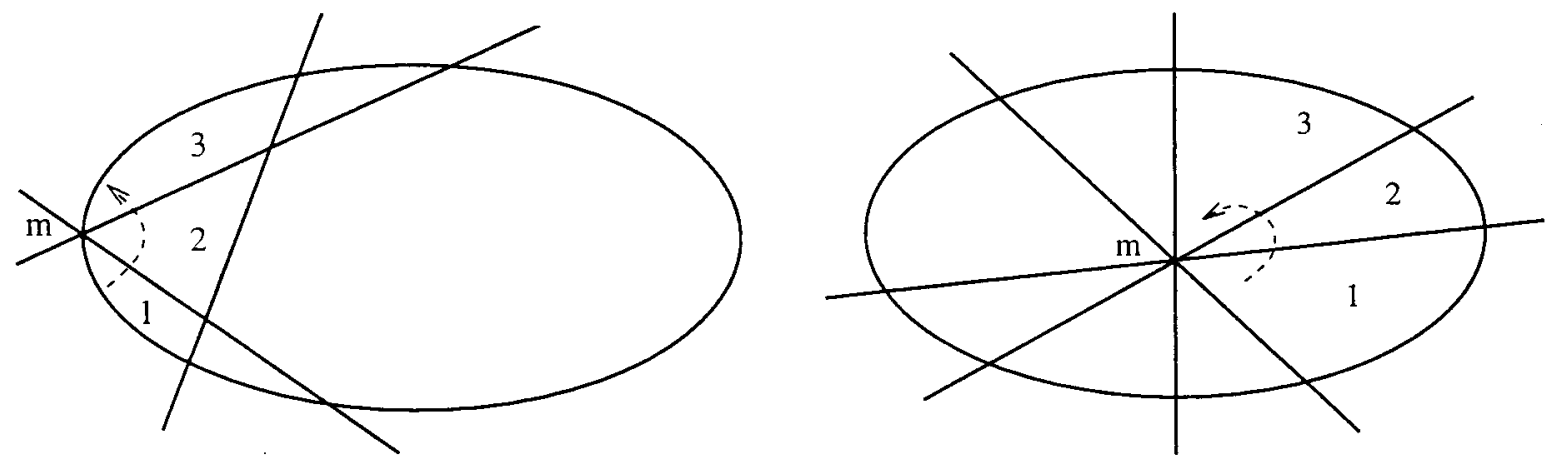

Fig. 2.5. $m=m(\Omega)$ or $m \in$ int $\Omega$. 
Case 2. $m \in \partial \Omega \backslash\{m(\Omega)\}$. The cells $\{T \in \mathcal{T}: m(T)=m\}$ are ordered clockwise if $m=\min \left(\Omega \cap \xi_{m}\right)$ and counterclockwise if $m=\max \left(\Omega \cap \xi_{m}\right)$, where $\xi_{m}$ is the vertical line through $m$ (see Fig. 2.6).
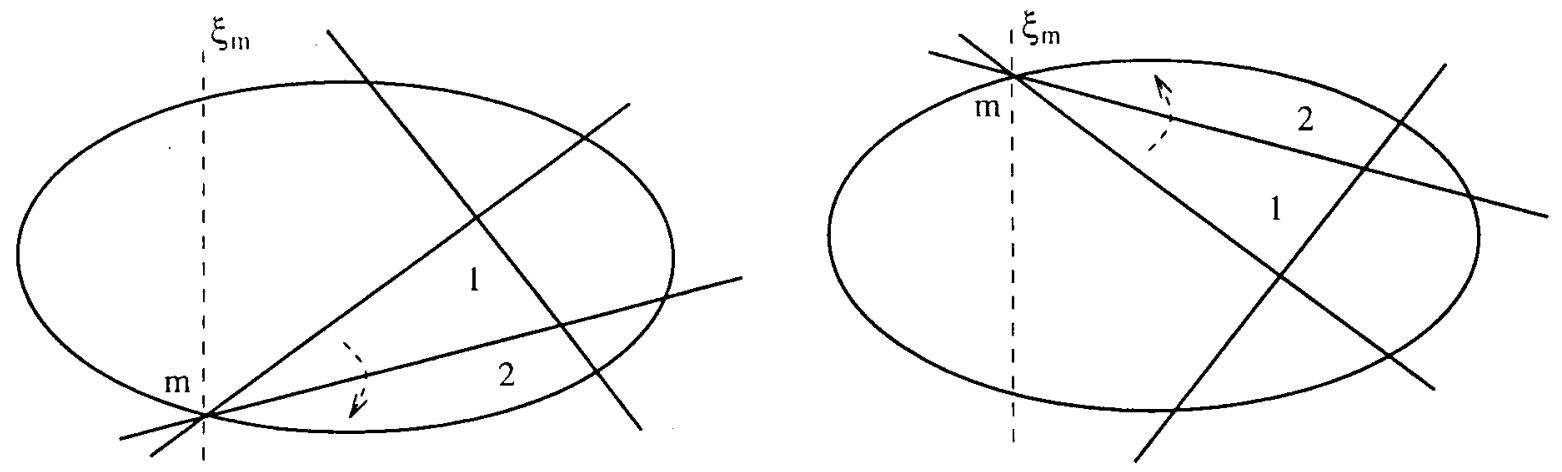

Fig. 2.6. $m \in \partial \Omega \backslash\{m(\Omega)\}$.

Thus, a total order for the elements of $\mathcal{T}$ has been defined, and we can write

$$
\mathcal{T}=\left\{T_{1}, \ldots, T_{n}\right\}, \quad \text { where } T_{i}<T_{i+1}, i=1, \ldots, n-1 .
$$

We now assign a type $\mathrm{A}, \mathrm{B}$ or $\mathrm{C}$ to each cell $T_{i}$ and then choose interpolation points on $T_{i}$ 's according to their types. Namely, $T_{1}$ is the only cell of type $A$ (since $\left.m\left(T_{1}\right)=m(\Omega)\right), T_{i}$ is a cell of type $B$ if $m\left(T_{i}\right) \in \partial \Omega \backslash\{m(\Omega)\}$, and a cell of type $C$ if $m\left(T_{i}\right) \in \operatorname{int} \Omega$. We put interpolation points of type A (as in Fig. 2.1) on $T_{1}$. It is easy to see that if $T_{i}$ is of type $\mathrm{B}$, then

$$
I_{i}:=\partial T_{i} \cap \partial\left(\bigcup_{j=1}^{i-1} T_{j}\right)
$$

is a nondegenerate line segment with an endpoint at $m\left(T_{i}\right)$. We choose interpolation points of type B on $T_{i}$, as in Fig. 2.2, where $T_{i} \subset \Omega_{1}, \bigcup_{j=1}^{i-1} T_{j} \subset \Omega_{0}, I_{i} \subset l$.

Let now $T_{i}, T_{i+1}, \ldots, T_{k}$ be a family of cells of type $\mathrm{C}$ with the same minimal point $m \in$ int $\Omega$, so that $m\left(T_{i-1}\right)<m, m\left(T_{i}\right)=m\left(T_{i+1}\right)=\cdots=m\left(T_{k}\right)=m$, $m\left(T_{k+1}\right)>m$. Then $\bigcup_{j=1}^{k} T_{j}$ is a subset of a cone, and we choose interpolation points of type $\mathrm{C}$ (as in Fig. 2.4, a) or b)) on it.

Theorem 2.2. [1] The set of all points chosen on the cells $T_{i}, i=1, \ldots, n$, as described above, is a Lagrange interpolation set for $S_{q}^{r}(\Delta)$.

We briefly describe the idea of the proof of Theorem 2.2 . Let $z_{1}, \ldots, z_{N}$ be all the points chosen on the cells in accordance with the above procedure. Then it follows from Theorem 2.1 that $N=\operatorname{dim} S_{q}^{r}(\Delta)$. Therefore, in order to prove that $\left\{z_{1}, \ldots, z_{N}\right\}$ is a Lagrange interpolation set for $S_{q}^{r}(\Delta)$ it is sufficient to check that for any $s \in S_{q}^{r}(\Delta)$,

$$
s\left(z_{1}\right)=0, \ldots, s\left(z_{N}\right)=0,
$$


implies

$$
s(z)=0, \text { for any } z \in \Omega .
$$

To this end we start from $T_{1}$ and see that (2.1) implies that

$$
s(z)=0, \quad \text { for any } \quad z \in T_{1}
$$

since $\left\{z_{1}, \ldots, z_{N}\right\} \cap T_{1}$ is a Lagrange interpolation set for $\widetilde{\Pi}_{q}$. Then we pass from $T_{2}$ to $T_{n}$ and see that for any $i \in\{2, \ldots, n\}$ such that $T_{i}$ is a cell of type $\mathrm{B}$,

$$
s(z)=0, \quad \text { for any } \quad z \in \bigcup_{j=1}^{i-1} T_{j} \quad \text { and } \quad z \in\left\{z_{1}, \ldots, z_{N}\right\} \cap T_{i}
$$

implies

$$
s(z)=0, \text { for any } z \in T_{i},
$$

since $\left\{z_{1}, \ldots, z_{N}\right\} \cap T_{i}$ is a Lagrange interpolation set for the corresponding space of truncated power functions $T_{q}^{r}$. Similarly, if $T_{i}, T_{i+1}, \ldots, T_{k}$ is a family of cells of type $\mathrm{C}$ with the same minimal point $m \in \operatorname{int} \Omega$, so that $m\left(T_{i-1}\right)<m, m\left(T_{i}\right)=$ $m\left(T_{i+1}\right)=\cdots=m\left(T_{k}\right)=m, m\left(T_{k+1}\right)>m$, then

$$
s(z)=0, \quad \text { for any } \quad z \in \bigcup_{j=1}^{i-1} T_{j} \quad \text { and } \quad z \in\left\{z_{1}, \ldots, z_{N}\right\} \cap \bigcup_{j=i}^{k} T_{j},
$$

implies

$$
s(z)=0, \quad \text { for any } \quad z \in \bigcup_{j=i}^{k} T_{j}
$$

since $\left\{z_{1}, \ldots, z_{N}\right\} \cap \bigcup_{j=i}^{k} T_{j}$ is a Lagrange interpolation set for the corresponding space of cone splines $K_{q}^{r}$. Thus, (2.2) follows by induction.

\section{Approximation Order of Bivariate Spline Interpolation}

In this section we consider spaces of bivariate splines with respect to special crosscut partitions $\Delta^{1}$ and $\Delta^{2}$. Let a rectangle $\Omega=[a, b] \times[c, d]$ and points $a=x_{0}<x_{1}<\cdots<x_{n_{1}}=b, c=y_{0}<y_{1}<\cdots<y_{n_{2}}=d$ such that $x_{i}-x_{i-1}=h_{1}, i=1, \ldots, n_{1} ; y_{j}-y_{j-1}=h_{2}, j=1, \ldots, n_{2}$, be given. We set $h=\max \left\{h_{1}, h_{2}\right\}$. By defining $R_{i, j}=\left[x_{i-1}, x_{i}\right] \times\left[y_{j-1}, y_{j}\right], i=1, \ldots, n_{1}$; $j=1, \ldots, n_{2}$, we obtain a partition of $\Omega$ into subrectangles $R_{i, j}$. If the diagonal from the lower left to the upper right vertex is added to each subrectangle $R_{i, j}$, then we denote the resulting partition by $\Delta^{1}$. If we add both diagonals to each subrectangle, then the resulting partition is denoted by $\Delta^{2}$.

Since both $\Delta^{1}$ and $\Delta^{2}$ are crosscut partitions, a basis for the spline spaces $S_{q}^{r}\left(\Delta^{\mu}\right), \mu=1,2$, is given in Theorem 2.1. Similarly, the application of Theorem 2.2 to $S_{q}^{r}\left(\Delta^{\mu}\right), \mu=1,2$, yields Lagrange interpolation sets of Nürnberger \& 
Riessinger $[13,14]$. We first describe these Lagrange interpolation sets for $S_{q}^{r}\left(\Delta^{1}\right)$. Then, by "taking limits", some Hermite interpolation sets are obtained, such that interpolation at them yields (nearly) optimal approximation order, under some restrictions on $r$ and $q$.

For constructing Lagrange interpolation sets for $S_{q}^{r}\left(\Delta^{1}\right), q \geq 4$, we describe four basic steps. For an arbitrary subtriangle $T$ of the partition $\Delta^{1}$, one of the following steps will be applied to $T$. (If the number of lines in Step $\mathrm{C}$ or $\mathrm{D}$ below is non-positive, then no points are chosen.)

Step A. (Starting step) Choose $q+1$ disjoint line segments $a_{1}, \ldots, a_{q+1}$ in $T$. For $i=1, \ldots, q+1$, choose $q+2-i$ distinct points on $a_{i}$.

Step B. Choose $q-r$ disjoint line segments $b_{1}, \ldots, b_{q-r}$ in $T$. For $i=1, \ldots, q-r$, choose $q+1-r-i$ distinct points on $b_{i}$.

Step C. Choose $q-2 r+\left[\frac{r}{2}\right]$ disjoint line segments $c_{1}, \ldots, c_{q-2 r+\left[\frac{r}{2}\right]}$ in $T$. For $i=$ $1, \ldots, q-2 r$, choose $q+1-r-i$ distinct points on $c_{i}$ and for $i=q-$ $2 r+1, \ldots, q-2 r+\left[\frac{r}{2}\right]$ choose $2(q-i)-3 r+1$ distinct points on $c_{i}$. (Here $[b]:=\max \{a \in \mathbb{Z}: a \leq b\}$.)

Step D. Choose $q-2 r-1$ disjoint line segments $d_{1}, \ldots, d_{q-2 r-1}$ in $T$. For $i=$ $1, \ldots, q-2 r-1$, choose $q-2 r-i$ distinct points on $d_{i}$.

Given a partition $\Delta^{1}$, we apply the above steps to the subtriangles of $\Delta^{1}$ as indicated in Fig. 3.2, where we choose horizontal, vertical and diagonal line segments as indicated in Fig. 3.1.

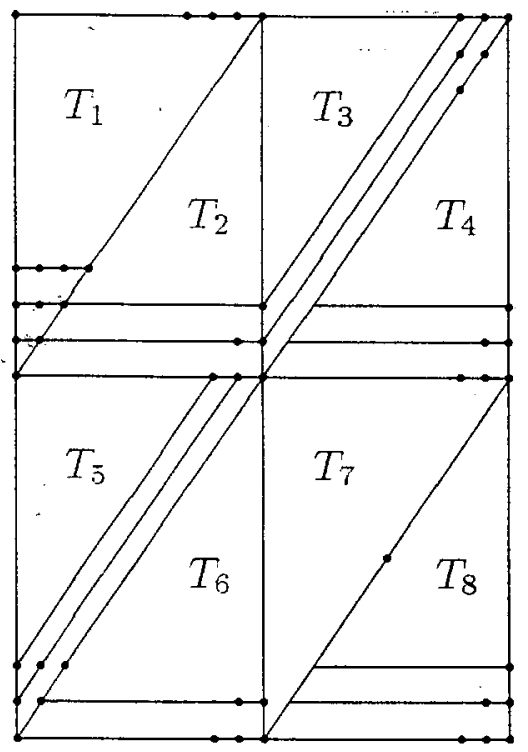

Fig. 3.1.

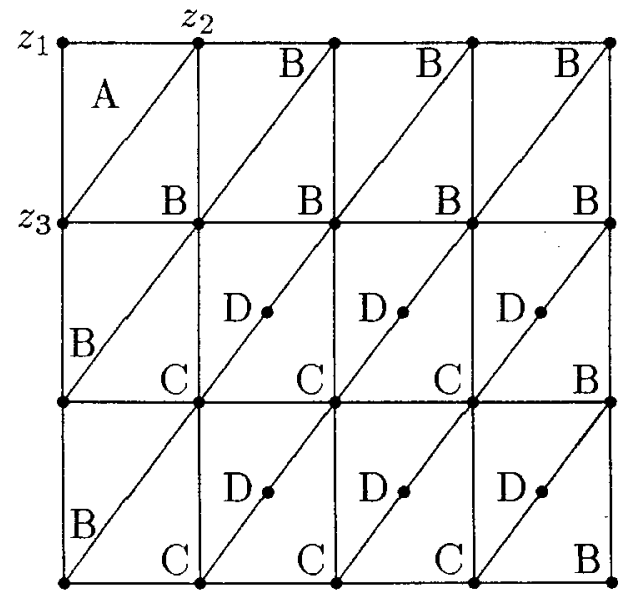

Fig. 3.2. 
The following construction of Hermite interpolation sets for $S_{q}^{r}\left(\Delta^{1}\right)$, which yields (nearly) optimal order approximation, was given by Nürnberger [12] for $r=1$ and by Davydov, Nürnberger \& Zeilfelder [8] for $r \geq 2$.

Let a sufficiently differentiable function $f \in C(\Omega)$ be given. In order to define Hermite interpolation conditions for a spline $s \in S_{q}^{r}\left(\Delta^{1}\right)$, where $q \geq 4$ if $r=1$, and $q \geq 3.5 r+1$ if $r \geq 2$, we describe four basic conditions. Let $T$ be an arbitrary subtriangle of the partition $\Delta^{1}$. If $T$ is not the first from the left triangle in the top row, then $\widetilde{T}$ denotes the adjacent subtriangle left of $T$ in the same row if it exists, and up of $T$ otherwise. We impose one of the following four conditions on the polynomial $p=\left.s\right|_{T} \in \widetilde{\Pi}_{q}$.

Condition A. (Starting condition) $D^{\omega} p(z)=D^{\omega} f(z), \omega=0, \ldots, q$, where $z$ is a vertex of $T$.

Condition B. $D^{\omega} p(z)=D^{\omega} f(z), \omega=0, \ldots, q-r-1$, where $z$ is the vertex of $T$ not belonging to $\widetilde{T}$.

Condition C. $p_{x^{\alpha} y^{\beta}}(z)=f_{x^{\alpha} y^{\beta}}(z), \alpha \geq 0, \beta \geq 0, \alpha+\beta \leq q-r-1, \alpha+2 \beta \leq$ $2 q-3 r-2$, where $z$ is the vertex of $T$ not belonging to $\tilde{T}$.

Condition D. $D^{\omega} p(z)=D^{\omega} f(z), \omega=0, \ldots, q-2 r-2$, where $z$ is the midpoint of the diagonal of $T$.

Note that while Conditions $\mathrm{A}, \mathrm{B}$ and $\mathrm{D}$ are symmetric with respect to $x$ and $y$, this is not the fact for Condition C. Fig. 3.3 presents the domain in which all integer points $(\alpha, \beta)$ should be taken in order to define Condition $\mathrm{C}$.

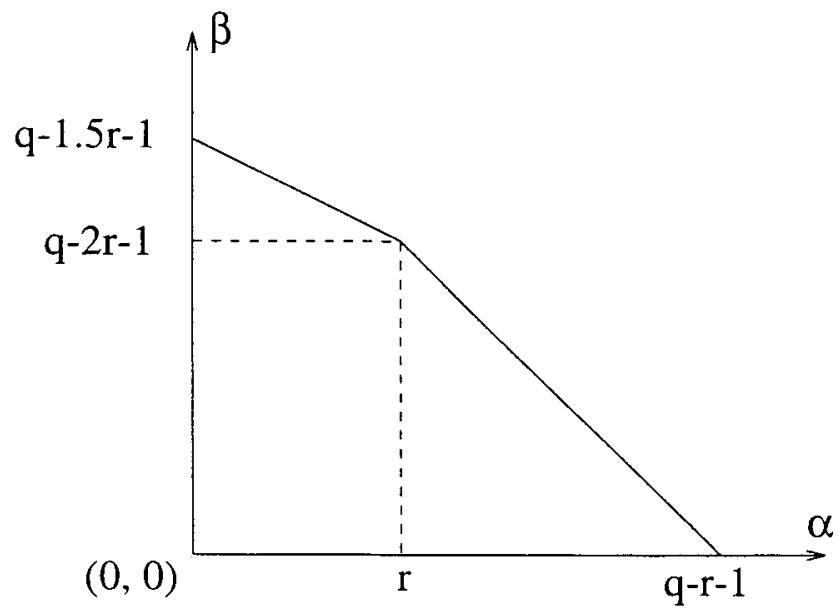

Fig. 3.3. Condition C.

Given a partition $\Delta^{1}$, the distribution of the Hermite interpolation conditions to the subtriangles is the same as for Lagrange interpolation and is indicated in Fig. 3.2.

In Theorems 3.1, 3.2 and 3.4 below, the norm denotes the maximum of the uniform norm over all subtriangles of the partition (w.r.t. the polynomial pieces). 
By using Bernstein-Bezier techniques, several authors proved results similar to those of Theorems 3.1 and 3.4 below for the special spline spaces $S_{3}^{1}\left(\Delta^{1}\right.$ ) (Sha [16]) and $S_{2}^{1}\left(\Delta^{2}\right)$ (Chui \& He [4], Sha [17], Zedek [18]). Moreover, Jeeawock-Zedek [9] proved that interpolation by $S_{3}^{1}\left(\Delta^{2}\right)$ yields approximation order two.

Theorem 3.1. [12] For each function $f \in C^{q+1}(\Omega)$, there exists a constant $K>0$ such that for the unique spline $s \in S_{q}^{1}\left(\Delta^{1}\right)$ which satisfies the above Hermite interpolation conditions, the following statements hold: For all $i \in\{0, \ldots, \rho-1\}$, $\left\|D^{i}(f-s)\right\| \leq K h^{\rho-i}$, where $\rho=4$ if $q=4$, and $\rho=q+1$ if $q \geq 5$. (The constant $K>0$ depends on $\left\|D^{q+1} f\right\|$ and is independent of $h$.)

Theorem 3.2. [8] Let integers $r \geq 2$ and $q \geq 3.5 r+1$ be given. For each function $f \in C^{q+1}(\Omega)$, there exists a constant $K>0$ such that for the unique spline $s \in S_{q}^{r}\left(\Delta^{1}\right)$ which satisfies the above Hermite interpolation conditions,

$$
\left\|D^{i}(f-s)\right\| \leq K h^{q+1-i}, \quad i=0, \ldots, q .
$$

(The constant $K>0$ depends on $\left\|D^{q+1} f\right\|$ and is independent of $h$.)

In view of Theorem 3.2 it is interesting to note that the approximation order of the spline space $S_{q}^{r}(\Delta)$ is optimal (i.e., $q+1$ ), if $q \geq 3 r+2$ (see de Boor \& Höllig [2], Chui, Hong \& Jia [5], and Lai \& Schumaker [10]). On the other hand, it was proved by de Boor \& Jia [3] that this is not true, if $q<3 r+2$, even for the $\Delta^{1}$-partition.

Remark 3.3. The method of proof in [12] can be applied to spline spaces $S_{q}^{1}\left(\widetilde{\Delta}^{1}\right)$, $q \geq 5$, where $\widetilde{\Delta}^{1}$ is a "deformation" of partition $\Delta^{1}$, by which we mean an arbitrary rectilinear embedding of the same triangulation in $\mathbb{R}^{2}$, as in Fig. 3.4. Under some restrictions on the angles between adjacent edges, corresponding Hermite interpolation scheme possesses optimal approximation order $h^{q+1}$, where $h$ is the maximal sidelength of the subtriangles.

We now describe in a similar way the construction of Lagrange and Hermite interpolation sets for $S_{q}^{1}\left(\Delta^{2}\right)$ (Results for $S_{q}^{r}\left(\Delta^{2}\right), r \geq 2$, are not yet available). As above, it turns out that interpolation at these points yields (nearly) optimal approximation order.

For constructing Lagrange interpolation sets for $S_{q}^{1}\left(\Delta^{2}\right), q \geq 2$, we again describe four basic steps.

Step A. (Starting step) Choose $q+1$ disjoint line segments $a_{1}, \ldots, a_{q+1}$ in $T$. For $i=1, \ldots, q+1$, choose $q+2-i$ distinct points on $a_{i}$.

Step B. Choose $q-1$ disjoint line segments $b_{1}, \ldots, b_{q-1}$ in $T$. For $i=1, \ldots, q-1$, choose $q-i$ distinct points on $b_{i}$.

Step C. Choose $q-3$ disjoint line segments $c_{1}, \ldots, c_{q-3}$ in $T$. For $i=1, \ldots, q-3$, choose $q-2-i$ distinct points on $c_{i}$.

Step D. Choose $q-2$ disjoint line segments $d_{1}, \ldots, d_{q-2}$ in $T$. For $i=1, \ldots, q-2$, choose $q-i$ distinct points on $d_{i}$. 


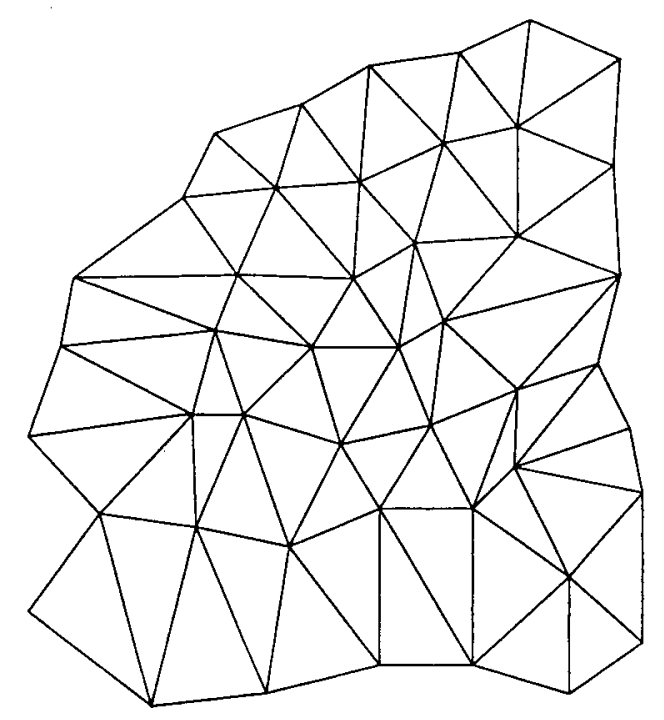

Fig. 3.4. An example of partition $\widetilde{\Delta}^{1}$.

Given a partition $\Delta^{2}$, we apply the above steps to the subtriangles of $\Delta^{2}$ as indicated in Fig. 3.6, where we choose horizontal, vertical and diagonal line segments as indicated in Fig. 3.5.

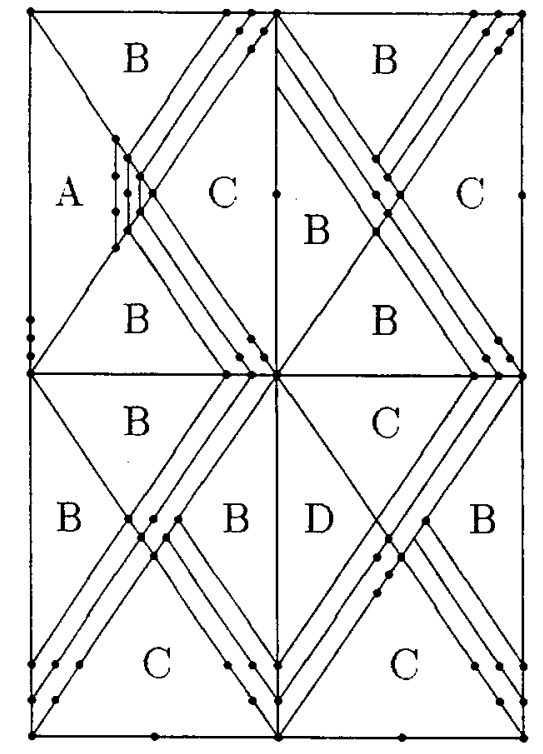

Fig. 3.5.

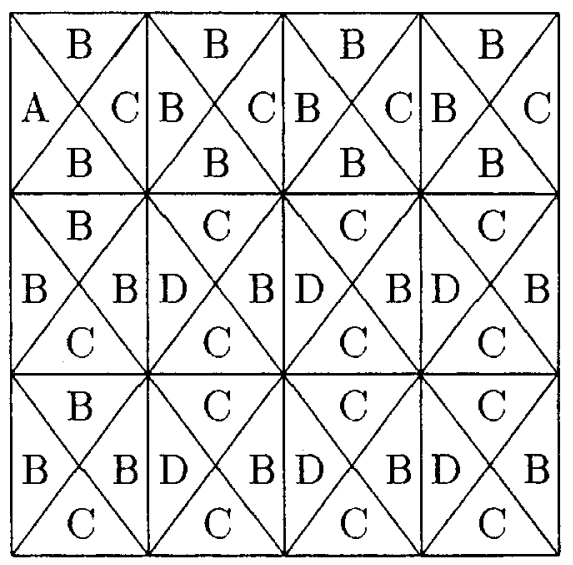

Fig. 3.6.

The following construction of Hermite interpolation sets for $S_{q}^{1}\left(\Delta^{2}\right)$, which differs in some parts from the one described above for $\Delta^{1}$, was given by Nürnberger \& Walz [15]. 
Let a sufficiently differentiable function $f \in C(\Omega)$ be given. We again have to describe four basic conditions. Let $T$ be an arbitrary subtriangle of the partition $\Delta^{2}$. We impose one of the following four conditions on the polynomial $p=\left.s\right|_{T} \in$ $\widetilde{\Pi}_{q}$, where $z$ is a vertex resp. a midpoint of an edge of $T$ as described below (cf. also Fig. 3.7).

Condition A. (Starting condition) $D^{\omega} p(z)=D^{\omega} f(z), \omega=0, \ldots, q$, where $z$ is a vertex of $T$.

Condition B. $D^{\omega} p(z)=D^{\omega} f(z), \omega=0, \ldots, q-2$, where $z$ is a vertex of $T$ not adjacent to the subtriangles already considered, e.g. $z_{4}$ or $z_{6}$.

Condition C. $D^{\omega} p(z)=D^{\omega} f(z), \omega=0, \ldots, q-4$, where $z$ is the midpoint of the edge of $T$ which is not adjacent to the subtriangles already considered, e.g. $z_{5}$.

Condition D. $p_{\varrho^{\alpha} \bar{\varrho}^{\beta}}(z)=f_{\varrho^{\alpha} \bar{\varrho}^{\beta}}(z), \alpha \geq 0, \beta \geq 0, \alpha+\beta \leq q-2, \beta \neq q-2$, where $\varrho=\left(\varrho_{1}, \varrho_{2}\right)$ is the unit vector in direction of the diagonal of $\Delta^{1}$, and $\bar{\varrho}=\left(-\varrho_{1}, \varrho_{2}\right)$, and where $z$ is the crossing point of the two diagonals in one subrectangle, e.g. $z_{7}$.

Given a partition $\Delta^{2}$, we apply the above steps to the subtriangles of $\Delta^{2}$ as indicated in Fig. 3.7. Note that the configuration of Hermite conditions is different from that of Lagrange conditions (cp. Fig. 3.6 and Fig. 3.7).

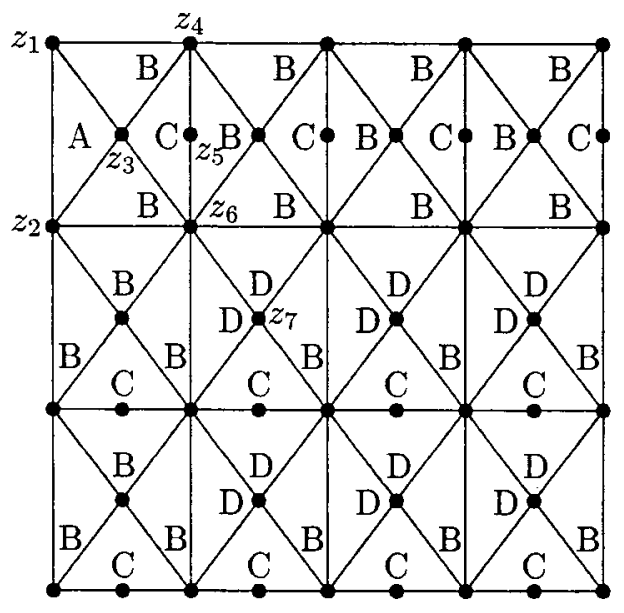

Fig. 3.7.

Theorem 3.4. [15] For each function $f \in C^{q+1}(\Omega)$, there exists a constant $K>0$ such that for the unique spline $s \in S_{q}^{1}\left(\Delta^{2}\right)$ which satisfies the above Hermite interpolation conditions, the following statements hold: For all $i \in\{0, \ldots, \rho-1\}$, $\left\|D^{i}(f-s)\right\| \leq K h^{\rho-i}$, where $\rho=q$ if $q \in\{2,3\}$, and $\rho=q+1$ if $q \geq 4$. (The constant $K>0$ depends on $\left\|D^{q+1} f\right\|$ and is independent of $h$.)

We briefly mention that these results can also be used for fitting of scattered data by using a two-step method, originally developed in [12]. The method is as 
follows: Let a (possibly non-rectangular) domain $\Omega$, points $w_{i} \in \Omega$ and corresponding data $f_{i}$ be given. In the first step, we approximate the data $f_{i}$ by any local method, e.g. interpolation by a piecewise polynomial $\widetilde{s}$ of degree $q$ such that $\|f-\tilde{s}\|=O\left(h^{q+1}\right)$ if $f_{i}=f\left(w_{i}\right)$ and $f \in C^{q+1}(\Omega)$. In general, piecewise polynomial interpolation is a simpler problem than spline interpolation and in any case, this is always possible if the data is regularly distributed over $\Omega$. In the second step, we interpolate the resulting function $\widetilde{s}$ (which may not even be continuous) by a smooth spline $s$ as described in this paper. As in Theorems 3.1, 3.2 and 3.4, it can be shown that $\left|f_{i}-s\left(w_{i}\right)\right|=O\left(h^{q}\right)$ or $O\left(h^{q+1}\right)$. More details on this and several numerical examples can be found in [12] and [15].

Acknowledgements. The research of O.V.Davydov was supported by the Alexander von Humboldt Foundation, under Research Fellowship.

\section{References}

1. Adam M. H., Bivariate Spline-Interpolation auf Crosscut-Partitionen, Doctoral Thesis, Mannheim 1996.

2. de Boor C. and Höllig K., Approximation power of smooth bivariate pp functions, Math. Z. 197 (1988), 343-363.

3. de Boor C. and Jia, Q., A sharp upper bound on the approximation order of smooth bivariate pp functions, J. Approx. Theory 72 (1993), 24-33.

4. Chui C. K. and He T. X., On location of sample points in $C^{1}$ quadratic bivariate spline interpolation, in Numerical Methods of Approximation Theory, ISNM 81, Collatz L., Meinardus G. and Nürnberger G. (eds.), 30-43, Birkhäuser, Basel, 1987.

5. Chui C. K., Hong D. and Jia Q., Stability of optimal-order approximation by bivariate splines over arbitrary triangulations, Trans. Amer. Math. Soc. 347 (1995), 3301-3318.

6. Chui C. K. and Wang R. H., Multivariate spline spaces, J. Math. Anal. Appl. 94 (1983), 197-221.

7. Dahmen W., Micchelli C. A., Recent progress in multivariate splines, in Approximation Theory IV, Chui C. K., Schumaker L. L. and Ward J. D. (eds.), 27-121, Academic Press, New York, 1983.

8. Davydov O. V., Nürnberger G. and Zeilfelder F., Approximation order of bivariate spline interpolation for arbitrary smoothness, submitted for publication.

9. Jeeawock-Zedek F., Interpolation scheme by $C^{1}$ cubic splines on a non uniform type-2 triangulation of a rectangular domain, C. R. Acad. Sci. Paris Sér. I Math. 314 (1992), 413-418. 
10. Lai M.-J. and Schumaker L., On the approximation power of bivariate splines, preprint.

11. Nürnberger G., Approximation by Spline Functions, Springer, Berlin, 1989.

12. Nürnberger G., Approximation order of bivariate spline interpolation, J. Approx. Theory 87 (1996), 117-136.

13. Nürnberger G. and Riessinger Th., Lagrange and Hermite interpolation by bivariate splines, Numer. Funct: Anal. Optim. 13, (1992), 75-96.

14. Nürnberger G. and Riessinger Th., Bivariate spline interpolation at grid points, Numer. Math. 71 (1995), 91-119.

15. Nürnberger G. and Walz G., Error analysis in bivariate spline interpolation, submitted for publication.

16. Sha Z., On interpolation by $S_{3}^{1}\left(\Delta_{m, n}^{1}\right)$, Approx. Theory Appl. 1 (1985), 71-82.

17. Sha Z., On interpolation by $S_{2}^{1}\left(\Delta_{m, n}^{2}\right)$, Approx. Theory Appl. 1 (1985), 1-18.

18. Zedek $F$., Interpolation de Lagrange par de splines quadratique sur un quadrilatere de $\mathbb{R}^{2}$, RAIRO Model. Math. Anal. Numer. 26 (1992), 575-593.

Oleg V. Davydov

Department of Mechanics and Mathematics

Dnepropetrovsk State University

pr. Gagarina 72

Dnepropetrovsk, GSP 320625, Ukraine

davydov@euklid.math.uni-mannheim.de

Günther Nürnberger, Guido Walz, Frank Zeilfelder

Lehrstuhl für Mathematik IV

Universität Mannheim

D-68131 Mannheim, Germany

\{nuernberger $\}\{$ walz $\}$ zeilfelder $\}$ @math.uni-mannheim.de 